\title{
Comparación entre el análisis 2-D y el Método de la Densidad de Fuerzas (discreto) para el equilibrio en estructuras de membrana
}

\author{
The 2-D continuous analysis versus the Density Force \\ Method (discrete) for structural membrane equilibrium
}

$\underline{\text { G. Viglialoro }}^{(*)}$, J. Murcia(**), F. Martínez ${ }^{(* * *)}$

RESUMEN

Este trabajo analiza el problema del equilibrio de una membrana y propone una comparación entre el conocido Método de la Densidad de Fuerzas, discreto y unidimensional (1-D), que aproxima la superficie de la membrana mediante una red espacial de cables, y el método continuo y bidimensional (2-D) sobre la propia superficie. Aunque el Método de la Densidad de Fuerzas representa una estrategia práctica y útil en el diseño de estructuras de membrana, se comprobará que el análisis continuo bidimensional no solo es más preciso y general sino que es más fiable, especialmente en aquellos casos en los que la membrana es el mismo tablero de una estructura portante (por ejemplo, una pasarela). En particular, una vez resumido el Método de la Densidad de Fuerzas, se planteará el problema continuo del equilibrio de membrana. A continuación se definirá un proceso de comparación entre ambos métodos, analizándolo por medio de ejemplos concretos. Finalmente, se señalarán algunas conclusiones.

445-27

Palabras clave: Membrana; pasarela; ecuaciones de equilibrio; aproximación 1-D discreta; análisis 2-D contínuo.

\section{SUMMARY}

This paper deals with the equilibrium problem of a membrane, presenting a comparison between the well-known 1-D discrete Force Density Method, using spatial cable networks as membrane surface approximations, and the 2-D continuous analysis of such a surface. Although Force Density is a practical and powerful method in structural membrane design, it will be checked that the 2-D continuous analysis is not only more accurate and general but necessary for new structural membrane applications such as footbridges. In this way, once summarized the discrete Density Force Method, the continuous approach is presented. Then, a comparison process between both methods is proposed, being developed for specific membrane examples. Finally, some conclusions are pointed out.

Keywords: Membrane; Footbridge; equilibrium equations; 1-D discrete approahc; 2-D continuous analysis.

\footnotetext{
(*) Universidad de Cádiz, (España).

${ }^{(* *)}$ Instituto de Ciencias de la Construcción Eduardo Torroja (CSIC), Madrid (España).

(***) Universidad Politécnica de Cataluña, Barcelona (España).

Persona de contacto/Corresponding author: giuseppe.viglialoro@uca.es (G. Viglialoro)
}

Recibido/Received: 21 jun 2011 Aceptado/Accepted: 03 oct 2012 Publicado online/ Published online: 13 jun 2013 


\section{NOTACIONES}

- Si $f(x, y)$ es una función de dos variables, utilizaremos la siguiente convención:

$$
\left\{\begin{array}{c}
f_{, 1}=f_{, x}=\frac{\partial f}{\partial x} \text { y } f_{, 2}=f_{, y}=\frac{\partial f}{\partial y} \\
f_{, 11}=f_{, x x}=\frac{\partial^{2} f}{\partial x^{2}} \text { y } f_{, 22}=f_{y y}=\frac{\partial^{2} f}{\partial y^{2}} \\
f_{, 12}=f_{, x y}=\frac{\partial^{2} f}{\partial x \partial y}=\frac{\partial^{2} f}{\partial y \partial}=f_{y x}=f_{, 21}
\end{array}\right.
$$

- Si $T:=\tau_{\alpha \beta}(\alpha=1,2$ y $\beta=1,2)$ es un tensor del segundo orden, utilizaremos la siguiente convención:

$$
\left\{\begin{aligned}
\tau_{11} & =\tau_{x x} \\
\tau_{12}=\tau_{x y} & =\tau_{y x}=\tau_{21} \\
\tau_{22} & =\tau_{y y}
\end{aligned}\right.
$$

$-\operatorname{div}(T \cdot \nabla f)$ es la divergencia del vector $T \cdot \nabla f$ y se define como $\sum_{\beta=1}^{2} \sum_{\alpha=1}^{2}\left(\tau_{\alpha \beta} f_{\alpha}\right)_{\beta}$

- Si $M$ es una matriz, $M^{T}$ indica su traspuesta

\section{INTRODUCCIÓN}

La idea de usar estructuras de membrana en aplicaciones portantes como las pasarelas (1) justifica el empleo de métodos de diseño y cálculo ajustados, mediante el análisis de la membrana tomándola como superficie y no con otra aproximación; esto es, un análisis continuo bidimensional (2-D). En efecto, con relación a otras, tales aplicaciones no solo suponen mayor responsabilidad estructural de uso y mayores esfuerzos (cargas de uso, alto pretensado y formas rebajadas de la membrana) sino que también requieren de la precisa definición previa de los esfuerzos de pretensado, asociados a la adecuada rigidez estructural en la fase de servicio.

Por otro lado, el Método de la Densidad de Fuerzas ha representado y representa un procedimento muy práctico y útil para el diseño y el cálculo de estructuras de membrana. Es un método discreto, unidimensional (1-D), basado en aproximar la membrana por una red de cables y considerar su equilibrio (2) (3). Entonces, actuando de modo estratégico, al suponer conocida a priori la relación entre la tracción y la longitud de cada cable, el planteamiento del equilibrio conduce a un sistema lineal que proporciona la posición de los puntos (nodos de la red) que definen la membrana.

En lo referente al análisis continuo 2-D, en el contexto ya citado, recordando que solo hay tracciones, la membrana se identifica a una superficie de curvatura gaussiana ne- gativa y sus esfuerzos a un tensor positivo de segundo orden. El equilibrio se expresa directamente por medio de ecuaciones en derivadas parciales, una de las cuales involucra a productos entre variables asociadas a la forma de la membrana y al tensor de esfuerzos ((4) para los detalles). Así, para un tensor de esfuerzos dado, la forma de la membrana representa la incógnita del problema; y viceversa.

Con relación al análisis 2-D, el Método de la Densidad de Fuerzas, además de lo que supone la propia aproximación 1-D en sí, tiene una limitación: no se pueden definir y controlar a priori las tracciones de los cables que modelan los esfuerzos de la membrana. Este aspecto es relevante en aplicaciones como las pasarelas, donde importa la definición previa y precisa de los esfuerzos de pretensado de la membrana.

En la literatura, el análisis de las membranas se ha centrado bastante en lo referente al problema de la búsqueda de forma de la membrana, con el empleo de diversos métodos 1-D y 2-D basados en técnicas estáticas o dinámicas para su resolución (ver, por ejemplo, (5) (6) (7) y (8) para análisis 1-D y (9) (10) para 2-D). En general, estos métodos pasan por un proceso de minimización de energía que lleva, a través de la resolución de un sistema en términos de desplazamientos nodales, a una cierta configuración de equilibrio de la membrana, que proporciona justamente su forma final. Así, está claro que la membrana se deforma.

Sin embargo la forma de la membrana puede obtenerse de un modo más directo, empleando únicamente las ecuaciones de equilibrio. En particular, como se ha visto, los procedimientos presentados al principio, uno 2-D y el otro 1-D, se basan en el análisis del equilibrio de la membrana; esto es, solo en términos de sus ecuaciones de equilibrio, sin introducir ningún tipo de deformación. Por tanto, parece coherente establecer un paralelismo entre estos dos métodos de análisis.

Por otro lado, mientras el método 1-D se usa para obtener la forma de la membrana, el análisis continuo 2-D es general, con una casuística (4) ajena a estas páginas.

Así, el objetivo principal de este trabajo es la comparación de ambos y, por tanto, su punto de partida es el problema del equilibrio de una membrana con el fin de conocer su forma. En la comparación, como se verá, se intenta extraer al límite, aunque de modo consistente, la información que proporcionan los resultados 1-D. 


\section{ECUACIONES DE EQUILIBRIO EN LA MEMBRANA}

En esta sección trataremos las ecuaciones de equilibrio de una membrana. Se empezará por resumir y analizar el método discreto de la Densidad de Fuerzas (1-D) y, a continuación, se presentará y estudiará el correspondiente problema continuo (2-D).

\subsection{Solución discreta 1-D}

El Método de la Densidad de Fuerzas se basa en considerar la membrana como una red de cables. Propuesto por Linkwitz y Scheck en el 1971 (2) (11), el método utiliza una simple estrategia matemática que transforma las ecuaciones de equilibrio de los nodos en un conjunto de ecuaciones lineales de fácil resolución, que permiten conocer la posición de los nodos, esto es, la forma de la membrana.

Resumamos el método. Una vez elegido un cierto grafo, red o malla espacial que permite solo conocer las conexiones entre los nodos que definen la membrana, se incluyen las condiciones de contorno, fijando algunos de los nodos del grafo, y se impone el equilibrio en todos los nodos. La Figura 1 muestra un ejemplo concreto en el cual las posiciones de los nodos 1, 2, 3, 4 han sido previamente establecidas; la incógnita del problema es la posición final del nodo 5, en el que se supone actúa una fuerza externa $f_{5}=\left(f_{5 x}, f_{5 y}, f_{5 x}\right)$.

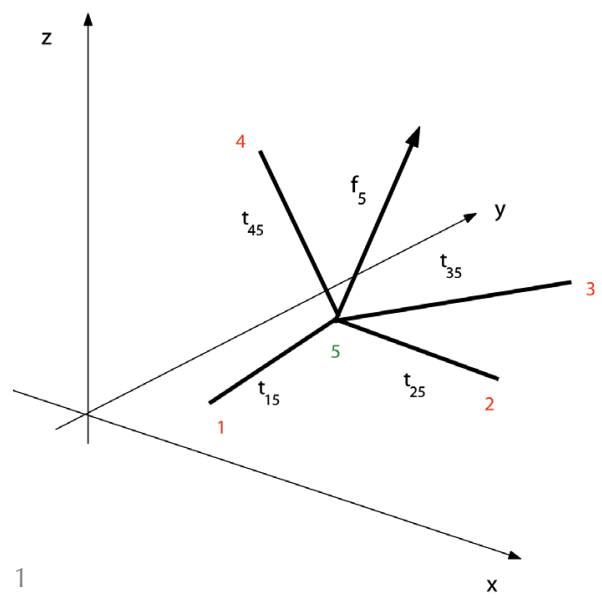

Si $t_{i 5}$ indica la tracción a lo largo del cable $i_{5}(i=1,2,3,4)$, las ecuaciones de equilibrio a lo largo de la dirección $x$ para el nodo $i$ son [1]

[1] $\quad \sum_{j=1}^{n} \frac{t_{i j}}{l_{i j}}\left(x_{i}-x_{j}\right)=f_{i,}$

siendo [2]

[2] $l_{i j}=\sqrt{\left(x_{i}-x_{j}\right)^{2}+\left(y_{i}-y_{j}\right)^{2}+\left(z_{i}-z_{j}\right)^{2}}$

Las longitudes $l_{i j}$ que aparecen en el denominador dependen de las posiciones noda- les, que son las incógnitas; esto hace que el sistema definido anteriormente sea no lineal. Es posible linealizar estas ecuaciones introduciendo para cada elemento las densidades de fuerzas [3]

$$
q_{i j}=\frac{t_{i j}}{l_{i i}},
$$

cuyos valores, que han de conocerse, se fijan a priori.

Para una estructura general de $n$ nodos y $b$ elementos (cables, barras, etc.), se considera la matriz de incidencia $C$ compuesta por $b$ filas y $n$ columnas. Esta matriz describe la conectividad de la estructura y queda definida de la siguiente manera: si un elemento une los nodos $i$ y $j$, entonces la correspondiente fila tiene +1 en la columna $i$ y -1 en la columna $j$. Tras esto, las ecuaciones de equilibrio a lo largo de la dirección $x$ se escriben, entonces, como [4]

$$
C^{T} Q C x=f_{x},
$$

siendo $Q$ la matriz diagonal $b \times b$ que contiene las densidades de fuerza, $x$ es el vector columna que recoge las coordenadas $x_{i}$ y $f_{x}$ es el vector columna que recoge las componentes en la dirección $x$ de las fuerzas nodales.

De la misma forma, pueden escribirse ecuaciones similares en términos de las coordenadas $y$ y $z$.

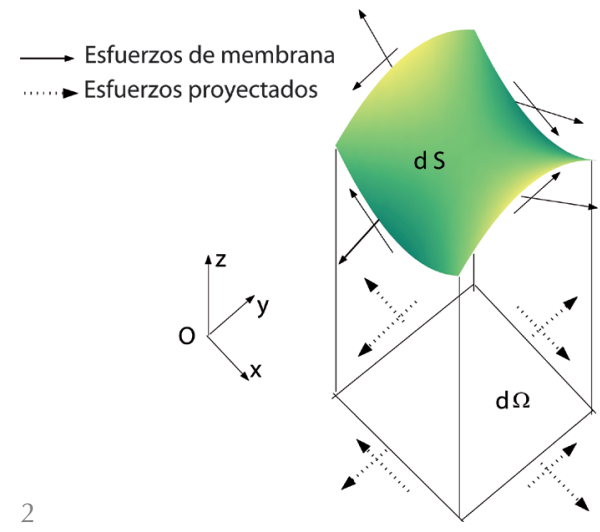

$\mathrm{Si}$, además, se fijan las coordenadas de $k$ (0 $<k<n)$ nodos, $C$ puede representarse como $C=\left[C_{u} C_{f}\right]$, donde el subíndice $u$ indica la matriz correspondiente a los nodos desconocidos y $f$ se refiere a la matriz de los fijados. En esta última expresión $C_{u}$ (o bien $C_{f}$ ) es una matriz $b \times(n-k)$ (o bien $b \times k$ ) obtenida escogiendo $b$ filas y $n$ - $k$ (o bien $k$ ) columnas de $C$; a partir de ello, la ecuación [4] puede escribirse como [5]

$$
C_{u}^{T} Q C_{u} x_{u}=f_{x}-C_{u}^{T} Q C_{f} x_{f},
$$

donde $C_{u}$ y $C_{f}$, y $x_{u}$ y $x_{f}$ representan las matrices de incidencia y los vectores columna relativos a los nodos incógnitos y conocidos, respectivamente.
1. Ejemplo de red de cables (grafo) para el Método de la Densidad de Fuerzas.

2. Membrana y tensores de esfuerzos. 
3. Tensor de esfuerzos proyectados.

4. Proyecciones verticales del tensor de esfuerzos $\tilde{N}_{\alpha \beta}$.
La solución de la ecuación [5] para la dirección $x$, junto con las correspondientes ecuaciones en las direcciones y y $z$, proporcionan el conjunto de todas las coordenadas nodales del grafo, es decir la forma final de la membrana.

Como se justificará en la sección 2.2., al plantear este problema de equilibrio para conocer la forma de la membrana en la fase de pretensado, las cargas externas se toman nulas; esto implica que el sistema anterior se simplifica en el siguiente [6]

$$
C_{u}^{T} Q C_{u} x_{u}=-C_{u}^{T} Q C_{f} x_{f} .
$$

En una estructura formada por cables a tracción todas las densidades de fuerzas son positivas, esto es $q_{i j}>0$. Así, $C_{u}^{T} Q C_{u}$ representa una matriz definida positiva, y por tanto, invertible; por consiguiente, el problema [4] siempre proporciona solución única.

\subsection{Solución continua 2-D}

Siguiendo la misma línea del trabajo (4), identifiquemos la membrana con una superficie $S$ de curvatura gaussiana negativa, gráfica de una función de dos variables $z=z(x, y)$ definida en un dominio $\Omega \subset \mathrm{R}^{2}$ (véase la Figura 2, que representa un elemento diferencial $d S$ de superficie y su proyección $d \Omega$ ). La Figura 3 muestra el plano tangente (aproximación local del elemento $d S$ ) y el tensor de esfuerzos, ambos proyectados en el plano $x O y$.

De nuevo, se trata de obtener la forma de la membrana en la fase de pretensado. El peso de la membrana es muy bajo y así, para tensiones de pretensado muy altas como aquí se supone, es relativamente insignificante y puede ser despreciado. De esta forma, tal y como se hizo en la sección 2.1., no se considerará ninguna carga externa.

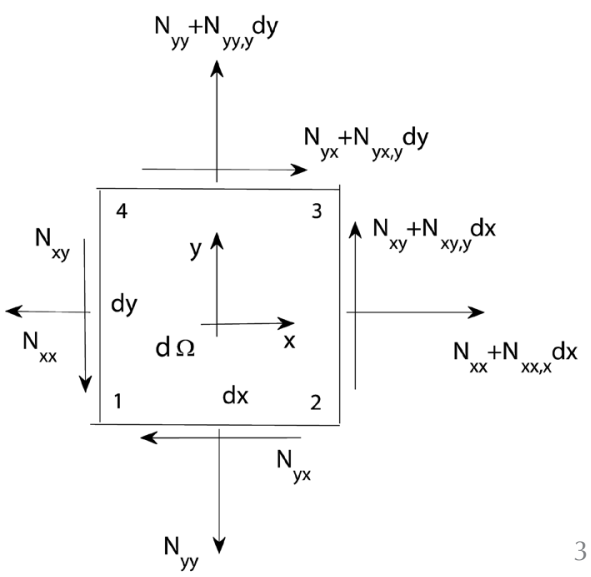

Si $N_{\alpha \beta}(\alpha, \beta=1,2)$ indica el tensor de esfuerzos de membrana proyectados (esto es, fuerza por unidad de longitud), las ecuaciones de equilibrio en el plano horizontal se resumen en el sistema [7]:
[7] $\quad\left\{\begin{array}{l}N_{x x, x}+N_{x y, y}=0 \\ N_{x y, x}+N_{y y, y}=0\end{array}\right.$

Como se muestra en la Figura 4, las expresiones de las componentes verticales de los esfuerzos de membrana $\tilde{N}_{\alpha \beta}$ pueden calcularse en términos de $N_{\alpha \beta}$ y de las pendientes de la superficie $S$. Entonces, el equilibrio vertical se expresa como [8]:

[8] $\quad\left(N_{x x} z_{x}+N_{y x} z_{y}\right)_{x}+\left(N_{x y} z_{x}+N_{y y} z_{y}\right)_{y}=0$

esto es [9]

$$
\begin{aligned}
& z_{, x}\left(N_{x x, x}+N_{x y, y}\right)+z_{y}\left(N_{x y, x}+N_{y y, y}\right) \\
& +N_{x x} z_{x x}+2 N_{x y} z_{x y}+N_{y y} z_{y y}=0
\end{aligned}
$$

Usando el sistema [7], la ecuación [9] se simplifican de modo que el equilibrio en términos de $N_{\alpha \beta}$ se escribe como [10] (4):

$$
\left\{\begin{array}{c}
N_{x x, x}+N_{x y, y}=0 \\
N_{x y, x}+N_{y y, y}=0 \\
N_{x x} z_{x x}+2 N_{x y} z_{x y}+N_{y y} z_{y y}=0
\end{array}\right.
$$

Siempre en el trabajo (4), las mismas ecuaciones de equilibrio se obtienen también a partir directamente de los esfuerzos de membrana $\tilde{N}_{\alpha \beta}$ (asimismo, fuerza por unidad de longitud); en efecto, usando la relación entre los esfuerzos $N_{\alpha \beta}$ y los esfuerzos de membrana $\tilde{N}_{\alpha \beta}$, es decir [11]:

[11] $\left\{\begin{array}{l}N_{y y}=\tilde{N}_{y y} \sqrt{1+z_{, x}^{2}} \cos \beta=\tilde{N}_{y y} \frac{\sqrt{1+z_{, x}^{2}}}{\sqrt{1+z_{, y}^{2}}}, \\ N_{x x}=\tilde{N}_{x x} \sqrt{1+z_{y}^{2}} \cos \alpha=\tilde{N}_{x x} \frac{\sqrt{1+z_{, y}^{2}}}{\sqrt{1+z_{, x}^{2}}}, \\ N_{x y}=\frac{\sqrt{1+z_{, y}^{2}}}{\sqrt{1+z_{, y}^{2}}} \tilde{N}_{x y}=\frac{\sqrt{1+z_{, x}^{2}}}{\sqrt{1+z_{, x}^{2}}} \tilde{N}_{y x}=N_{y x},\end{array}\right.$

(ver Figuras 2 y 4), se obtiene el mismo sistema [10].

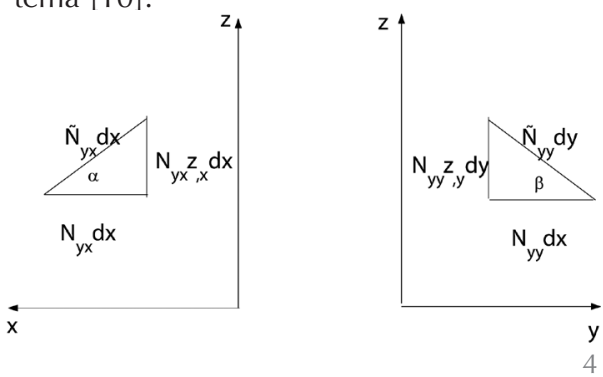

Tras ello, fijado el tensor (positivo) de esfuerzos queda por determinar la forma de la membrana.

\section{Formulación del problema continuo}

Sea $\sigma:=N_{\alpha \beta}$ un tensor de esfuerzos positivo y simétrico definido en un dominio $\Omega \subset R^{2}$, tal que [12]:

$$
\begin{gathered}
\left\{\begin{array}{c}
N_{x x, x}+N_{x y, y}=0 \\
N_{x y, x}+N_{y y, y}=0
\end{array} \leftrightarrow \sum_{\beta=1}^{2} N_{\alpha \beta, \beta}=0\right. \\
\alpha=1,2
\end{gathered}
$$


Sea, además, $g$ la función definida sobre el borde $\Gamma=\partial \Omega$ de $\Omega$ cuyos valores fijan la forma del borde espacial de la membrana: hallar la superficie $z=z(x, y)$ definida en $\Omega$, de manera tal que [13]:

\section{[13]}

$z_{x x} N_{x x}+2 z_{x y} N_{x y}+z_{y y} N_{y y}=\operatorname{div}(\sigma \cdot \nabla z)=0$ en $\Omega$, $z=g$ sobre $\Gamma$.

Se puede demostrar que este problema tiene una única solución, estable con respecto a eventuales perturbaciones de los datos de contorno. De hecho el sistema [10] es un problema elíptico con condiciones de Dirichlet, cuya solución está caracterizada por ser la función que minimiza un cierto funcional energético (ver los detalles en (4)).

En general, no es posible minimizar por medio de expresiones analíticas dicho funcional; por lo cual es preciso recurrir a métodos numéricos, discretizando el continuo, que proporcionan soluciones aproximadas.

En particular, utilizando el Método de los Elementos Finitos (ver por ejemplo (12) y (13)), una vez establecida una malla para $\Omega$, indiquemos con $n_{t}$ el número de nodos total en $\Omega$ y $n_{b}$ el número de nodos del borde $\Gamma$. Utilizando las aproximaciones [14].

$$
z \approx \sum_{j=1}^{n_{k}} z_{j} N_{j} \quad y \quad g \approx \sum_{j=1}^{n_{b}} g_{j} N_{j}
$$

$\left(N_{j}\right.$ representan las funciones de forma lineales definidas en $\Omega$ y $\Gamma$, respectivamente), la solución del sistema [10] es [15].

$$
z=G g \text {, }
$$

siendo $G$ la matriz simétrica obtenida invirtiendo

$$
\left[\begin{array}{cc}
K & A^{T} \\
A & 0
\end{array}\right]
$$

En las expresiones anteriores, $z=\left(z_{1}, z_{2}, \ldots, z_{n}\right)$ y $g=\left(g_{1}, g_{2}, \ldots, g_{n}\right)$ indican los valores nodales de $z$ y $g, K$ la matriz simétrica $\left(n_{t} \times n_{t}\right)$ de rigidez cuyos elementos se calculan por medio de las expresiones $K_{i j}=\int \nabla N_{i} \cdot \sigma \cdot \nabla N_{j} d \Omega$, $A$ la matriz $\left(n_{b} \times n_{t}\right)$ de los multiplicadores de Lagrange, que almacena los datos relativos a las condiciones de contorno (ver (12)), y 0 la matriz $\left(n_{b} \times n_{b}\right)$ nula.

\subsection{Algunas observaciones sobre los métodos continuo y discreto}

Es útil remarcar que el problema continuo 2-D determina la forma continua de la membrana, proporcionando la expresión de la superficie por medio de la fórmula [15], siempre y cuando se conozcan previamente la distribución de esfuerzos de membrana en cada punto así como su frontera espacial (condición de contorno). Como se ha comentado, la forma de la membrana es estable con los datos; más exactamente, a una pequeña variación de los datos de frontera (forma espacial del borde) corresponde una pequeña variación de la solución (forma general de la membrana).

Por lo contrario, el Método de la Densidad de Fuerzas determina la forma discreta de la membrana, proporcionando la posición espacial de todos sus nodos (fórmula [6]), siempre y cuando se conozcan las densidades de fuerzas relativas a cada cable que identifica la membrana así como los puntos fijos que definen el borde espacial de la misma (condición de contorno). Contrariamente a lo que ocurre en el enfoque bidimensional, se puede comprobar (ver (14)) que, en general, la solución es sensible con respecto a la variación de los datos de borde y que depende del tipo de grafo utilizado (triangular, cuadrangular, etc).

La Tabla 1 resume los dos enfoques, recogiendo para cada método los pasos correspondientes para su resolución. En particular, puede apreciarse que el método 2-D proporciona una relación directa entre esfuerzo y forma, al contrario de lo que ocurre para el método 1-D. En efecto, el enfoque discreto no permite definir a priori las tracciones de los cables, sino que pueden conocerse solo usando la fórmula [3], es

\begin{tabular}{|c|c|c|}
\hline Método & Método discreto 1-D & Método continuo 2-D \\
\hline Datos & $\begin{array}{c}\text { Grafo para } \Omega \\
\text { (grafo proyectado) } \\
\text { Densidades } q_{i j} \\
\text { Valores de borde } g_{i}\end{array}$ & $\begin{array}{c}\text { Malla para } \Omega \\
\text { Tensor } N \alpha \beta \\
\text { Función de borde } g\end{array}$ \\
\hline Solución & $\begin{array}{l}\text { Vectores de posición de los nodos } \\
x, y \text { y } z \text { dados por el sistema [6] }\end{array}$ & $\begin{array}{c}\text { Función continua } z \text { dada } \\
\text { por el sistema [15] }\end{array}$ \\
\hline Post proceso & $\begin{array}{l}\text { Tracciones de los cables [3]: } \\
\qquad l_{i j} \rightarrow t_{i j}=q_{i j} l_{i j}\end{array}$ & \\
\hline
\end{tabular}
decir, al finalizar el proceso.

Tabla 1. Esquema resumen para los métodos continuo y discreto.

\section{COMPARACIÓN ENTRE EL ANÁLISIS DEL EQUILIBRIO 1-D Y 2-D}

En esta sección se propone un proceso de comparación entre los dos tipos de análisis de equilibrio, discreto y continuo, previamente estudiados.

\subsection{Proceso de comparación}

Partiendo de la solución 1-D del Método de la Densidad de Fuerzas, se pretende extrapolarla de forma coherente en lo posible a una solución 2-D, con el objetivo de comparar la anterior con la que resulta directamente del método continuo 2-D.

Para ello, se seguirá el siguiente esquema:

I. Resolución de un problema de equilibrio de membrana a través de la apro- 
5. Red de cables (membrana) y tracciones (de los cables) proyectadas para el nodo $i$.

6. Fuerza en el nodo $i$ y definición del tensor de esfuerzos. ximación 1-D, en el caso de un grafo cuadrangular y considerando todas las densidades de fuerzas constantes, esto es, (solución del sistema [6]).

II. Definición punto a punto, en los nodos, de los esfuerzos proyectados, y, tomando en cuenta los valores de las tracciones obtenidos tras el apartado I (post proceso).

III. Aproximación continua en todos los puntos del dominio de los esfuerzos y, a partir de los valores puntuales definidos en el apartado II y de manera que verifiquen todas las propiedades del tensor continuo 2-D.

IV. Resolución del problema de equilibrio de membrana mediante el análisis continuo 2-D, tomando como tensor el definido en el apartado III y como condiciones de contorno las establecidas en el apartado I (solución del sistema [15]).

Sin duda alguna, la clave de este proceso es definir adecuadamente los esfuerzos $N_{a \beta}$ punto a punto en términos de las tracciones $t_{i j}$ (apartado II), calculadas por resolución del problema 1-D (apartado I).

Observemos las Figuras 5 y 6 , que identifican el equilibro 3-D del nodo $i$ para las tracciones de los cables $A, B, C$ y $D$ (color negro) que aproximan la membrana, y el mismo equilibrio para las tracciones proyectadas $a=\left(a_{x}, a_{y}\right), \quad b=\left(b_{x}, b_{y}\right), \quad c=\left(c_{x}, c_{y}\right)$ y $d=\left(d_{x}, d_{y}\right)$, en color azul.

Vamos a considerar, por ejemplo, el esfuerzo $N_{x x}$. Teniendo siempre en cuenta la relación $t_{i j}=l_{i j}$ y siendo el esfuerzo longitudinal $N_{x x}$ la fuerza en la dirección $x$ dividida por la longitud del lado en el cual esta actúa, es natural la siguiente definición: [16]

$$
N_{x x}=\frac{a_{x}+c_{x}}{b_{y}+d_{y}}
$$

Razonando de la misma forma para los demás esfuerzos $N_{y y}, N_{x y}$ y $N_{y x}$, es natural definir el tensor de esfuerzos puntuales $N_{\alpha \beta}$ en términos de las tracciones proyectadas de los cables por medio de estas expresiones: [17]

$$
\left\{\begin{array}{l}
N_{x x}=\frac{a_{x}+c_{x}}{b_{y}+d_{y}} \\
N_{y y}=\frac{b_{y}+d_{y}}{a_{x}+c_{x}} \\
N_{x y}=\frac{a_{y}+c_{y}}{b_{y}+d_{y}} \\
N_{y x}=\frac{b_{x}+d_{x}}{a_{x}+c_{x}}
\end{array}\right.
$$

En general, los valores $N_{x y}$ y $N_{y x}$ obtenidos en [17] difieren entre sí. Para obtener la simetría necesaria, se tomará el valor medio $\left(N_{x y}+N_{y x}\right) / 2$ para $N_{x y}$ y $N_{y x}$.
Con el objetivo de proporcionar una distribución continua coherente de los esfuerzos $N_{\alpha \beta}$, se consideran polinomios que aproximen los valores nodales previamente definidos. Por supuesto, como estos polinomios pueden no verificar todas las propiedades del tensor $N_{\alpha \beta}$ (apartado III), se operará fijando una aproximación para $N_{x x}$ y hallando las expresiones de los esfuerzos restantes mediante la integración de las relaciones: [18]

$$
\sum_{\beta=1}^{2} N_{\alpha \beta, \beta}=0 \quad(\alpha=1,2) .
$$

Tras todo ello, se puede resolver el problema continuo por medio del paso IV.

\subsection{Algunos ejemplos del proceso de comparación}

Vamos a desarrollar el proceso definido en la sección 3.1. por medio de algunos ejemplos. Tal y como se ha analizado en detalle en el artículo (4), el problema continuo definido por las ecuaciones [12] y [13] está bien planteado; en particular el método numérico empleado en el mismo trabajo proporciona una aproximación muy coherente con respeto a la solución exacta, también en los casos en que la malla utilizada no sea excesivamente fina. Debido a todo ello, tomaremos como solución de referencia la obtenida por el método 2-D, y la denotaremos con $z_{\text {con }}$. De esta forma, es posible definir el error relativo $e$ como: [19]

$$
e=\frac{\left|z_{\text {con }}-z_{\text {dis }}\right|}{\left|z_{\text {con }}\right|}
$$

siendo $z_{\text {dis }}$ la solución correspondiente al problema discreto (ecuación [6]).
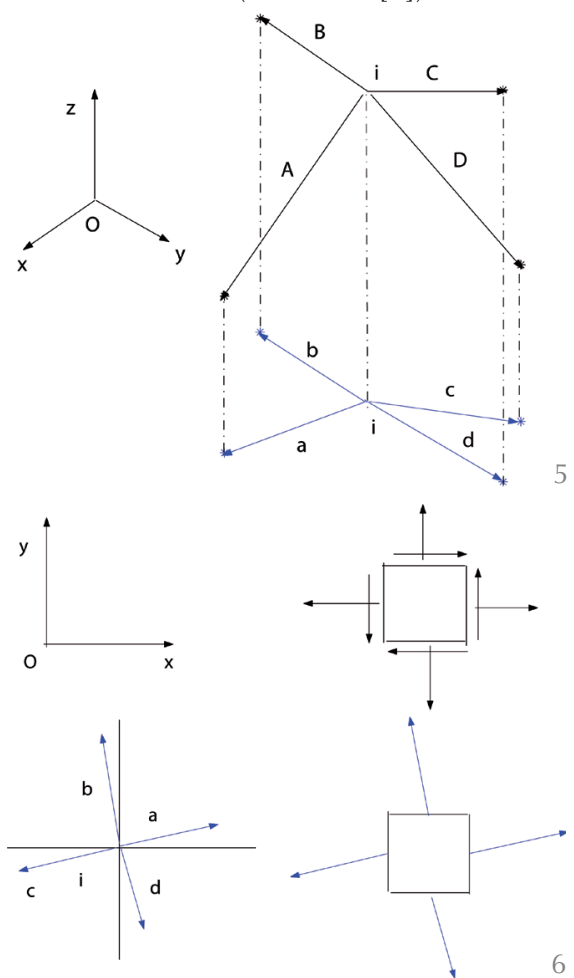
Para entender mejor la comparación propuesta y poder obtener resultados en parte realistas, se analizarán dos ejemplos, definidos respectivamente en un dominio rectangular y otro no rectangular. El segundo ejemplo, con bordes laterales cóncavos, más propio de las pasarelas de membrana con cables laterales de borde, se asemeja, en particular, al del prototipo real descrito y analizado en la referencia (1).

Conviene subrayar que, en dichos ejemplos, las dimensiones de $t_{i j}, N_{\alpha \beta}$ y las variables de longitud, no reflejadas en los resultados, deben ser coherentes; por ejemplo, $k N, k N / m$ y $m$. Además, con el fin de simplificar el análisis, nos centraremos en el comportamiento del error en el origen $O$ de los ejes.

En los ejemplos que siguen, tomaremos particiones del dominio plano definiendo en cada dirección una distribución de puntos del tipo $(2 k+1) \times(2 k+1)$, con $k=1,2,3$, $4,5 \ldots$; dicha partición, tal y como se analiza en la tesis (14), garantiza una convergencia rápida del método de la Densidad de Fuerzas.

\section{Ejemplo 1}

Dominio rectangular: $\mathrm{R}=[-5,5] \times[-2,2] \quad(\mathrm{Fi}-$ guras 7,8 y 9).

A. Forma de la membrana en el borde: parabólica y constante $\left(q_{i j}=1\right)$

Sea $z( \pm 5, y)=0,5 y^{2}$ y $z(x, \pm 2)=2$

Tomando para $R$ una partición del grafo del tipo $(2 k+1) \times(2 k+1)$, los esfuerzos obtenidos utilizando las expresiones del sistema [17] no dependen de la densidad de la malla (esto es, del valor elegido por $k$ ) y se mantienen constantes nodo por nodo para cualquier partición. En particular, los valores son $N_{x x}=2,5$; $N_{x y}=N_{y x}=0$ y $N_{y}=0,4$ en todos los puntos; por consiguiente la aproximación polinomial del apartado III no es necesaria.

En estas condiciones, utilizando la partición correspondiente a $k=8$, el valor numérico en el origen $O=(0,0)$ para la solución discreta $z_{\text {dis }}$ vale 1,1789. Por otro lado, resolviendo el problema bidimensional (2-D) utilizando los valores
7. Ejemplo 1-A: resultado gráfico de la solución.

a. Gráfica de la solución discreta. b. Gráfica de la solución continua.

8. Ejemplo 1-B: resultado gráfico de la solución.

a. Gráfica de la solución discreta.

b. Gráfica de la solución continua.

8. Ejemplo 1-C: resultado gráfico de la solución.

a. Gráfica de la solución discreta. b. Gráfica de la solución continua.
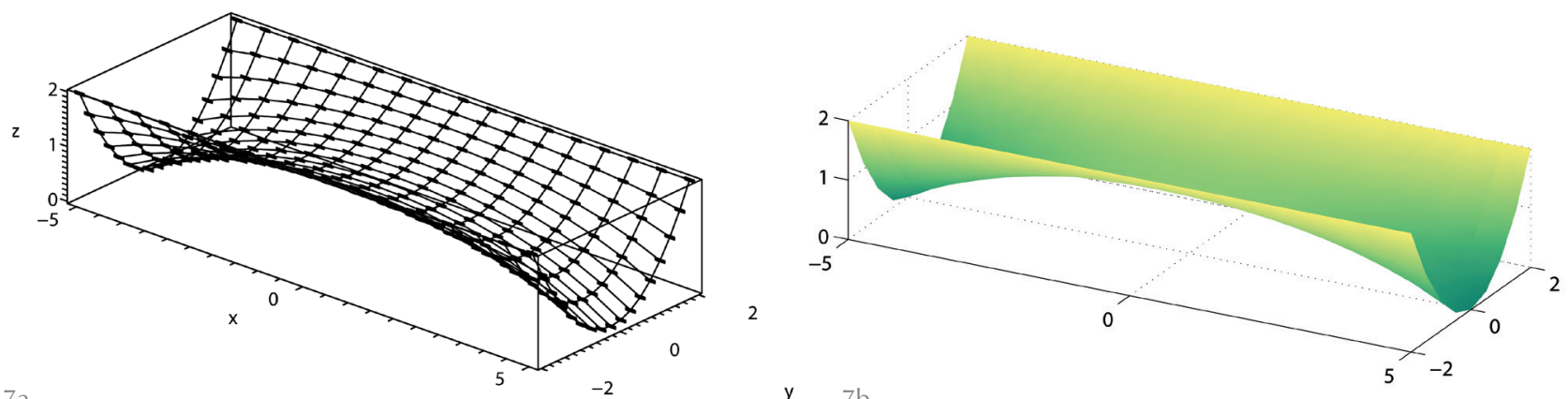

$7 a$
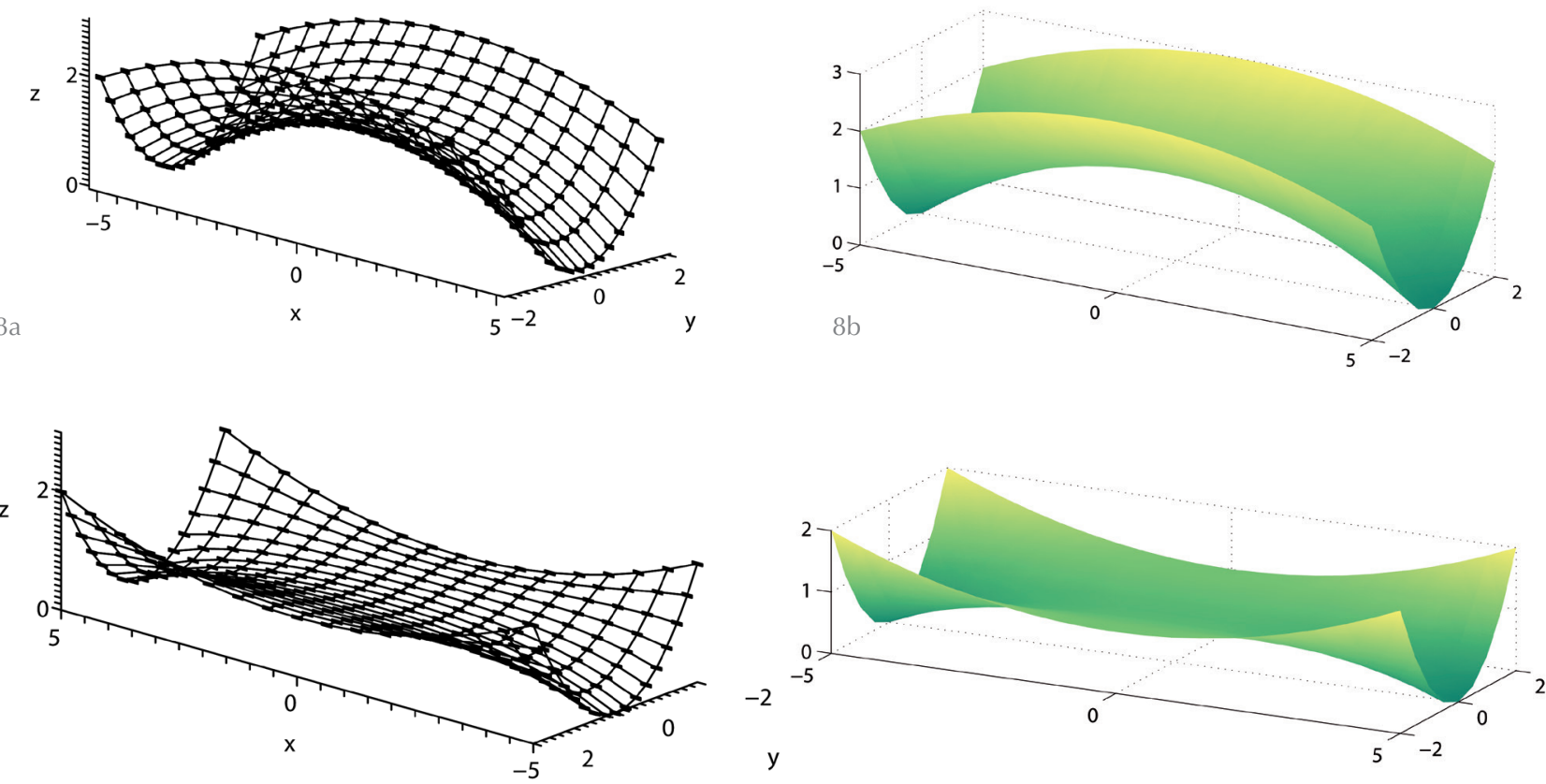
10. Malla cuadrangular de 289 nodos para un dominio no rectangular; caso $k=8$

11. Ejemplo 2: resultado gráfico de la solución.

a. Gráfica de la solución discreta.

b. Gráfica de la solución continua. calculados para los esfuerzos y las mismas condiciones de contorno, el valor en el origen de la solución $z_{\text {con }}$ vale 1,1826. De esta manera, el error relativo resulta ser $e=\left|z_{\text {con }}-z_{\text {dis }}\right| /\left|z_{\text {con }}\right|=8,6 \cdot 10^{-3}$. La Figura 7 muestra el resultado gráfico de la solución calculada por los dos métodos.

B. Forma de la membrana en el borde: parabólica y con curvaturas opuestas $\left(q_{i j}=1\right)$

Sea $z( \pm 5, y)=0,5 y^{2}$ y $z(x, \pm 2)=-0,004 x^{2}+3$

Operando de la misma forma que antes los valores de los esfuerzos obtenidos resultan ser $N_{x x}=2,5 ; N_{x y}=N_{y x}=0$ y $N_{y}=0,4$. Equivalentemente, en el origen $O, z_{\text {dis }}$ vale 1,5875 y $z_{\text {con }} 1,5897$, siendo el error relativo $e=\left|z_{\text {con }}-z_{\text {dis }}\right| /\left|z_{\text {con }}\right|=1,4 \cdot 10^{-3}$. La Figura 8 muestra el resultado gráfico de las soluciones.

C. Forma de la membrana en el borde: parabólica y con curvaturas iguales $\left(q_{i j}=1\right)$

Sea $z( \pm 5, y)=0,5 y^{2}$ y $z(x, \pm 2)=-0,004 x^{2}+1$

Operando de la misma forma que antes los valores de los esfuerzos obtenidos resultan ser $N_{x x}=2,5 ; N_{x y}=N_{y x}=0$ y $N_{y}=0,4$. Equivalentemente, en el origen $O, z_{\text {dis }}$ vale 0,7626 y $z_{\text {con }} 0,7755$, siendo el error relativo $e=\left|z_{\text {con }}-z_{\text {dis }}\right| /\left|z_{\text {con }}\right|=1,6 \cdot 10^{-2}$. La Figura 9 muestra el resultado gráfico de las soluciones.

Con relación al caso $\mathrm{C}$, mostrado en la Figura 9, conviene insistir en que la membrana mantiene su curvatura de Gauss negativa; a pesar de que, por la forma del contorno, parezca inducirse lo contrario en ciertas zonas.
Al contrario de lo que ocurre en un dominio rectangular, tomando para $\Omega$ una partición del grafo del tipo $(2 k+1) \times(2 k+1)$ los esfuerzos obtenidos por el sistema [17] no solo varían punto a punto sino que dependen de la compacidad de la malla. De esta forma, fijada la malla de elementos finitos de la Figura 10 para $\Omega$, correspondiente una vez más en el grafo al valor $k=8$, se calcula por aproximación una distribución continua de los valores de $N_{\alpha \beta}$. En este caso particular, el ajuste polinomial (de cuarto grado) que se utiliza proporciona estas expresiones aproximadas para los esfuerzos:

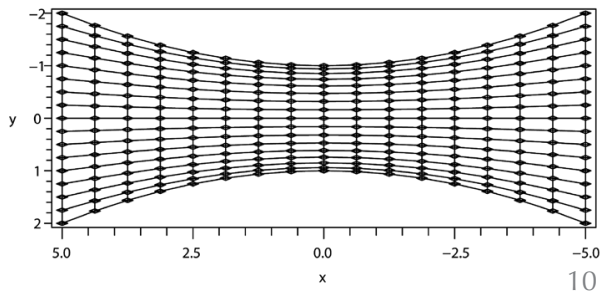

$N_{x x}=0,009 x^{4}+0,435 y^{4}-0,212 x^{2} y^{2}-0,214 x^{2}+2,87 y^{2}+3,932$

$$
\begin{aligned}
& N_{x y}=N_{y x}=-0,036 x^{3} y+0,141 y^{3}+0,428 x y, \\
& N_{y y}=0,054 x^{2} y^{2}-0,214 y^{2},
\end{aligned}
$$

que, como puede comprobarse, han sido justamente definidos de manera que cumplan todas las condiciones de equilibrio [20]

$$
\sum_{\beta=1}^{2} N_{\alpha \beta, \beta}=0
$$

Finalmente los valores de la solución $z_{\text {dis }}$ y $z_{\text {con }}$ en el origen $O=(0,0)$ valen 1,5875 y 1,4246 , respectivamente. El correspondiente error relativo, esta vez sensiblemente superior a los casos anteriores (algo más del $11 \%$ ), vale $e=\mid z_{\mathrm{con}^{-}}$ $z_{\text {dis }}|/| z_{\text {con }} \mid=0,114$. La Figura 11 muestra los resultados gráficos de las soluciones.

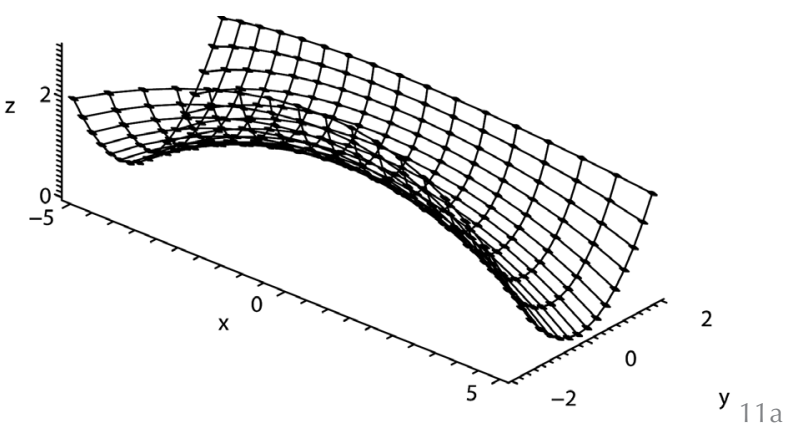

Ejemplo 2

Dominio no rectangular: $\Omega=\{-5 \leq x \leq 5 \wedge$ $-y(x) \leq y \leq y(x)\}$, siendo $y(x)=1+0,004 x^{2}$ (Figuras 10 y 11 ).

Forma de la membrana en el borde: parabólica y con curvaturas opuestas $\left(q_{i j}=1\right)$

Sea $z( \pm 5, y)=0,5 y^{2}$ y $z(x, \pm y(x))=$ $-0,004 x^{2}+3$.

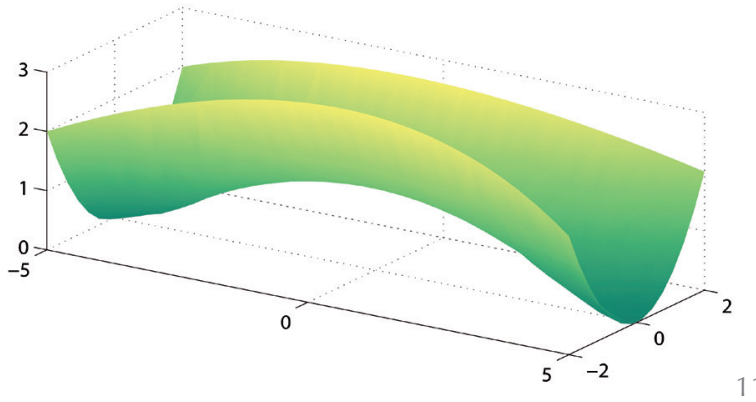

3.3. Análisis de los resultados obtenidos

Los ejemplos analizados muestran que el proceso de comparación propuesto devuelve errores significativos en problemas definidos sobre dominios generales (no rectangulares). Asociar estos errores únicamente a la compacidad de la malla y a la aproximación matemática usada para definir la distribución continua de los esfuerzos, que no puede ajustarse a los valores exactos de los mismos, es incorrecto. 
Existe una argumentación más profunda, íntimamente relacionada a la esencia del problema.

En efecto, debido a la geometría curvilínea del dominio, los valores de los esfuerzos (sistema [17]) cambian en términos de la refinación de la malla, de manera que no hay posibilidad de asociarle al problema un determinado tensor de esfuerzos; por consiguiente no queda definida una única estructura.

Por otro lado, el error entre las soluciones discretas y continuas es prácticamente nulo si se consideran dominios rectangulares, y esto es coherente con el proceso de comparación arriba planteado; profundicemos este comportamiento.

$\mathrm{R}=4 \times \mathrm{R}$

\begin{tabular}{|l|l|l|l|}
\hline$R$ & & & \\
& & 0 & $R$ \\
\hline & & & \\
\hline$\Omega$ & & & \\
\hline 12 & & & \\
\hline
\end{tabular}

La Figura 12 identifica un rectángulo con una partición de tipo $(2 k+1) \times(2 k+1)$. Es fácil comprobar que esta partición conserva las proporciones entre los lados que definen cada uno de los rectángulos que forman la malla. En efecto, a partir de las tracciones de los cables, el sistema [17] devuelve un tensor de esfuerzos diagonal $N_{\alpha \beta \prime}$ con coeficientes constantes, que verifica todas las propiedades del tensor continuo 2-D: [21] (ver Figura 13).

$$
\left\{\begin{array}{l}
N_{x x}=\frac{a_{x}+c_{x}}{b_{y}+d_{y}} \\
N_{y y}=\frac{b_{y}+d_{y}}{a_{x}+c_{x}} \\
N_{x y}=0
\end{array}\right.
$$

En consecuencia, el proceso de comparación proporciona una correspondencia bien precisa entre las tracciones de los cables propios del método 1-D y los esfuerzos de membrana del método 2-D, de modo que es posible asociar al problema un determinado tensor de esfuerzo; por consiguiente queda definida una única estructura.

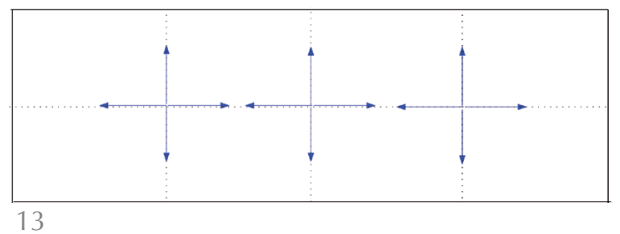

\section{ANOTACIONES Y CONCLUSIONES}

Este trabajo propone un procedimiento comparativo entre el Método de la Den- sidad de Fuerzas (análisis discreto 1-D) y el análisis continuo (2-D), ambos inherentes al problema de equilibrio de una membrana a tracción. Al referirse sendos problemas al cálculo y al diseño de estructuras de membrana exclusivamente a través de las correspondientes ecuaciones de equilibrio, la comparación se establece en términos de la forma de la membrana y de sus esfuerzos.

En primer lugar, es importante remarcar que una limitación fundamental del Método de la Densidad de Fuerzas reside en que su utilización no permite fijar los esfuerzos a priori, sino que los mismos, dependiendo de la forma final de la membrana, solo pueden calcularse cuando el proceso de resolución haya terminado. De esta manera, no es posible establecer una correspondencia directa entre los esfuerzos deseables para la membrana (previos al análisis) y la forma de esta.

Por el contrario, el análisis continuo 2-D implica un problema diferencial de contorno para un cierto tensor de esfuerzos dado, cuya solución representa la forma de la membrana, esta vez íntimamente relacionada con el tensor previamente fijado.

Por otra parte, el proceso de comparación parece reforzar la idea de que en general no es posible estudiar con precisión el equilibrio de un continuo bidimensional, como es una membrana, a través de una red de cables. En efecto, la forma hallada por el Método de la Densidad de Fuerzas depende de la malla empleada; si bien la comparación demuestra que este mismo método se ajusta perfectamente al relativo problema bidimensional en casos singulares definidos sobre dominios rectangulares (nada habituales, por otra parte, en estructuras de membrana y menos aún en aplicaciones como pasarelas).

En resumen, para el diseño y el cálculo de muchas estructuras de membrana el Método de la Densidad de Fuerzas sigue siendo indudablemente muy práctico y oportuno. Sin embargo, el método de análisis continuo 2-D aquí mostrado no solo ofrece una alternativa más general y precisa que la discreta, sino que también es la opción recomendable para el diseño y el cálculo de estructuras de membrana en las que es preciso controlar previamente los esfuerzos en todo punto. Todo ello concurre en aplicaciones portantes como las pasarelas, con gran responsabilidad estructural de uso, importantes esfuerzos y necesidad de definir de manera previa y ajustada los esfuerzos de pretensado.
12. Partición del tipo $(2 k+1) \times(2 k+1)$ para un dominio rectangular.

13. Dominio rectangular: las direcciones principales coinciden con los ejes. 


\section{REFERENCIAS}

(1) Murcia, J. (2007). Tecnología de pasarelas con estructura de membrana. Informes de la Construcción, 59(507): 21-31.

(2) Linkwitz, K. (1999). Form finding by the "direct approach" and pertinent strategies for the conceptual design of prestressed and hanging structures. International Journal of Space Structures, 14(2): 73-87.

(3) Linkwitz, K., Sheck, H. J. (1971). Einige Bemerkungen zur Berechnung von vorgespannten Seilnetzkonstruktionen. Ingenieur-Archiv, 40: 145-158. En alemán.

(4) Viglialoro, G., Murcia, J., Martínez, F. (2009). Problemas asociados al equilibrio en estructuras de membrana con bordes rígidos. Informes de la Construcción, 61(516): 57-66. doi: 10.3989/ic.08.038.

(5) Argyris, J. H., Angelopoulos, T., Bichat, B. (1974). A general method for the shape finding of lightweight tension structures. Computer Methods in Applied Mechanics and Engineering, 3: 135-149.

(6) Barnes, M. R. (1975). Applications of dynamic relaxations to the design and analysis of cable, membrane and pneumatic structures. Proc. Int. Conf. Space Struct., Guildford.

(7) Bathe, K. J. (1982). Finite Element Procedures in Engineering Analysis. Englewood Clis, NJ Prentice-Hall.

(8) Motro, R. (1984). Forms and forces in tensegrity systems. In Third International Conference on Space Structures; Guildford; pp. 180-185.

(9) Bonet, J., Mahaney, J. (2001). Form Finding of membrane structures by updated reference method with minimum mesh distortion. International Journal of Solids and Structures, 38(32-33): 5469-5480.

(10) Geymonat, G., Leger, A. (1999). Nonlinear Spherical Caps and Associated Plate and Membrane Problems. Journal of Elasticity, 57(3): 171-200.

(11) Sheck, H. J. (1974). The force density method for form finding and computation of general net works. Computational Methods in Applied Mechanics and Engineering, 3(1): 115-134.

(12) Hughes, T. J. R. (1987). The Finite Element Method. Linear Static and Dynamic Finite Element Analysis. Mineola, New York Dover Publications.

(13) Zienkiewicz, O. C., Taylor, R. L. (2000). The Finite Element Method. ButterworthHeinemann.

(14) Viglialoro, G. (2006). Análisis matemático del equilibrio en estructuras de membrana con bordes rígidos y cables. Pasarelas: forma y pretensado (Tesis doctoral). Universidad Politécnica de Cataluña. Enlace: http://www.tesisenxarxa.net/TDX-0515107-100745/

(15) Lewis, W. J. (2003). Tension Structures. Form and Behaviour. Edit. Thomas Telford. 